\title{
Real-World Treatment Patterns, Time to Next Treatment, and Economic Outcomes in Relapsed or Refractory Multiple Myeloma Patients Treated with Pomalidomide or Carfilzomib
}

\author{
Chi-Chang Chen, MSPharm, PhD; Kejal Parikh, MS; Safiya Abouzaid, PharmD, MPH; \\ Lea Purnomo, BSc; Catherine B. McGuiness, MA, MSc; Mohamed Hussein, MD; \\ and Rolin L. Wade, RPh, MS
}

\begin{abstract}
BACKGROUND: Negligible real-world evidence exists for later line treatment of multiple myeloma (MM) to assist treatment decisions or reimbursement models, such as episode-based payments.

OBJECTIVE: To describe the treatment patterns and clinical/economic outcomes when pomalidomide or carfilzomib is used for relapsed/refractory MM.

METHODS: A U.S. claims database was used to identify MM patients with an initial pomalidomide or carfilzomib claim (index date) between February 1, 2013, and February 28, 2015, which was assumed to be relapse therapy. Treatment regimens were defined as all MM chemotherapy observed within 60 days of index. Patients receiving pomalidomide and carfilzomib within 60 days of index were excluded. Time to next treatment (TTNT), a progression proxy, was defined as the addition of a new agent $>60$ days from index or as treatment restart following a >90-day therapy gap. Cost estimations used plan-allowed amounts. Descriptive statistics were used to compare outcomes between treatment groups, and regression models were used to adjust for baseline patient characteristics.
\end{abstract}

RESULTS: There were 454 patients initiating treatment with pomalidomide $(n=264)$ or carfilzomib $(n=190)$ during the index period. The most frequent initial regimens for pomalidomide patients included pomalidomide + dexamethasone (47.0\%) and pomalidomide alone (33.0\%); the most frequent regimens for carfilzomib patients were carfilzomib alone (45.3\%) and carfilzomib + dexamethasone (14.7\%). The most frequent next line treatment for pomalidomide patients who progressed was the addition of $(14.0 \%)$ or switch to $(15.0 \%)$ carfilzomib \pm dexamethasone and for carfilzomib patients, the most frequent next line treatment was pomalidomide + dexamethasone $(9.3 \%)$ and carfilzomib alone or carfilzomib + dexamethasone + cyclophosphamide (6.7\% each). The median (95\% CI) TTNT for pomalidomide patients was 11.9 (10.7-14.8) compared with 9.4 (7.7-10.0) months for carfilzomib $(P=0.060)$. For patients followed to progression (pomalidomide: $n=100,37.9 \%$; carfilzomib: $n=75,39.5 \%$ ), mean TTNT was longer for patients initiating therapy with pomalidomide (6.9 months) versus carfilzomib (5.3 months, $P=0.016)$. When adjusted for baseline confounders, pomalidomide patients had a nonsignificant longer time to a subsequent treatment line. Inpatient encounters observed during the index line were very low $($ mean $=1)$ for both groups; outpatient encounters were fewer in pomalidomide patients. Adjusted analyses revealed inpatient encounters were higher $(P=0.005)$, while outpatient use was lower in pomalidomide patients $(P=0.006)$. Unadjusted median costs incurred during the initial line were similar between the 2 groups (pomalidomide: $\$ 102,805$; carfilzomib: $\$ 127,203 ; P=0.110$ ) but significantly lower in pomalidomide patients after adjusting for baseline characteristics $(P=0.013)$. Unadjusted per patient per month (PPPM) costs incurred over the entire follow-up period were lower in pomalidomide-initiated patients $(\$ 18,298$ vs. $\$ 24,734, P=0.001)$ but not statistically significant in adjusted analyses $(P=0.230)$.
CONCLUSIONS: A longer time to a subsequent line of therapy was observed in pomalidomide patients compared with carfilzomib patients, although the difference lost significance in adjusted analyses. Compared with carfilzomib, pomalidomide patients were observed to have lower unadjusted median PPPM costs over the entire post-index period and lower adjusted mean monthly costs during initial therapy.

J Manag Care Spec Pharm. 2017;23(2):236-46

Copyright $\odot 2017$, Academy of Managed Care Pharmacy. All rights reserved.

\section{What is already known about this subject}

Recently approved therapies for relapsed/refractory multiple myeloma provide patients and providers with advanced options for care.

Real-world evaluations of treatment patterns and outcomes, including cost comparing newer treatment options such as pomalidomide or carfilzomib, are sparse.

New payment models for cancer care are evolving, with payers, providers, and other stakeholders looking for ways to address the high and increasing costs of cancer care.

\section{What this study adds}

The treatment patterns with pomalidomide or carfilzomib in later line therapy for multiple myeloma are described, including common regimens and time to next treatment progression.

Health care resource utilization and cost were found to be different between these therapies.

As the health care system moves away from fee-for-service models, this type of real-world data may help inform episode-based cancer treatment decisions.

$\mathrm{M}$ ultiple myeloma (MM) is a hematologic cancer formed by malignant plasma cells and is relatively uncommon, with a lifetime risk of 1 in $143(0.7 \%)$ in the United States. ${ }^{1}$ It is estimated that in 2016 about 30,330 new patients (17,900 men and 12,430 women) were diagnosed with MM, and 12,650 patients (6,430 men and 6,220 women) died from $\mathrm{MM}$ in the United States. ${ }^{1}$ The 5 -year survival rate for patients with MM is $47 \%$ and is dependent on individual patient 
characteristics. ${ }^{2}$ Survival rates are higher in younger populations ${ }^{1,2}$ and the earlier the disease is detected, the better chance a person has of surviving 5 years after being diagnosed..$^{1-3}$

The management of MM has changed over time. There has been a series of treatments that have had a positive effect on the survival time and quality of life of patients. ${ }^{4}$ Despite these advances, the disease remains incurable. Extending time to progression is often the main objective of treatment. ${ }^{4}$ Combinations of immunomodulating (IMiDs) regimens, such as lenalidomide, and proteasome inhibitors, such as bortezomib, are becoming widely used as first- and second-line therapy, along with corticosteroids (e.g., dexamethasone and prednisone).,5

In the last several years, new agents have been added to the list of treatment options for relapsed/refractory multiple myeloma (rrMM). Many of the recently approved regimens are a combination of 2 novel agents with corticosteroids (triplet therapy). At the time of this analysis, there were 2 new therapies approved for use alone or in combination with dexamethasone: pomalidomide + dexamethasone and carfilzomib monotherapy.

Pomalidomide, an IMiD agent approved in 2013 for rrMM in patients with previous use of bortezomib and lenalidomide, has shown efficacy in pretreated myeloma patients, the majority of whom were refractory to lenalidomide and bortezomib. ${ }^{6}$ The MM-003 phase 3 trial of pomalidomide+low-dose dexamethasone (LoDEX) versus high-dose dexamethasone in rrMM demonstrated a $31 \%$ response rate, ${ }^{7}$ with a median progression-free survival (PFS) of 4.0 months and a median overall survival of 12.7 months in pomalidomide+LoDEX patients. Of the treatment regimens recently approved for rrMM, pomalidomide + LoDEX is the only one with demonstrated improved survival versus the comparator arm (12.7 vs. 8.1 months; hazard ratio $[\mathrm{HR}]=0.74 ; 95 \%$ confidence interval $[\mathrm{CI}]=0.56-0.97 ; P=0.03) .^{7}$ At baseline, the majority of the sample ( $>90 \%$ in each group) had received more than 2 previous treatments, with a median of 5 previous treatments per group. Pomalidomide is orally administered and is well tolerated, with side effects including anemia, neutropenia, thrombocytopenia, upper respiratory tract infection, fatigue and weakness, constipation, shortness of breath, diarrhea, fever, back pain, and nausea. ${ }^{8}$ Pomalidomide can be used in patients with mild-tomoderate renal impairment (i.e., serum creatinine $\leq 3 \mathrm{mg} / \mathrm{dL}$ ), and neuropathy is rarely seen, although worsening of preexisting neuropathy has been reported. ${ }^{9}$

Carfilzomib is a selective proteasome inhibitor that was first approved for myeloma patients who did not respond to firstline thalidomide or lenalidomide therapy. ${ }^{10,11}$ An open-label, single-arm phase 2 study of patients received single-agent carfilzomib $\left(20 \mathrm{mg} / \mathrm{m}^{2}\right)$ to determine overall response rate after 6 cycles in bortezomib-naive adult rrMM patients having received at least 2 previous therapies, including lenalidomide, thalidomide, or stem cell transplant. The overall response rate was $47.6 \%$, with a time to progression of 8.3 months and a duration of response of 13.1 months. ${ }^{12}$ Another phase 3 study, FOCUS, randomized 315 heavily pretreated rrMM patients to single-agent carfilzomib or to best supportive care with a steroid and oral cyclophosphamide. The trial did not meet the primary endpoint, and no significant differences in overall survival were found between the 2 treatment arms. ${ }^{5}$

A large phase 3 trial, ASPIRE, randomized 792 patients and demonstrated that the addition of carfilzomib to lenalidomide and dexamethasone resulted in significantly improved PFS in rrMM patients. ${ }^{13}$ Carfilzomib was well tolerated, with adverse events including myelosuppression, fatigue, diarrhea, and suspected cardiac effects. Neuropathy was uncommon, and carfilzomib can be used in all degrees of renal insufficiency. ${ }^{6,9}$

While new treatment options in cancer care hold the promise of dramatic improvements in PFS and overall survival, the issue of high cost has not gone unnoticed by policymakers. ${ }^{14}$ Health care systems are evaluating alternatives to the fee-forservice model based on volume of care for new models such as episode-based or bundled payment systems. These policymakers will need real-world evidence to develop strategies and balance choices that link payment to quality care that benefits patients while making care more affordable. ${ }^{15}$ While payment methods were not tested in this study, the results focus on several elements of effectiveness and cost of care.

Because regimens with a single novel agent including pomalidomide or carfilzomib were newly approved for rrMM at the time of this analysis, we sought to describe their real-world treatment patterns and associated clinical and cost outcomes.

\section{Methods}

\section{Data Source}

This study was a retrospective cohort analysis using the PharMetrics Plus claims database, which contains adjudicated medical and pharmacy claims for more than 150 million health plan members across the United States from 2006 through March 2015, at the time of this study, and is representative of the U.S. commercially insured population. International Classification of Diseases, Ninth Revision, Clinical Modification (ICD-9-CM) codes were used to record data regarding inpatient and outpatient diagnoses. Other data elements recorded included inpatient and outpatient procedures and retail and mail-order prescription drug records. Costs as health planallowed amount were available for all services rendered, as were dates of service for all claims.

\section{Patient Selection}

Patients aged $\geq 18$ years on index with a claim for pomalidomide or carfilzomib between February 1, 2013, and February 28, 2015, were identified in the database, with the first observed claim serving as the index date. Patients were required to have at least 2 claims with a diagnosis of MM 
(ICD-9-CM codes 203 and 203.0x), at least 30 days apart, within the period of 6 months pre-index to 1 month postindex. Continuous enrollment was required $\geq 6$ months before and $\geq 3$ months after the index date. Patients with 2 or more diagnoses for other lymphomas and/or leukemias $\geq 30$ days apart during the study period (i.e., January 1, 2008, to March 31,2015) were excluded. Patients who received a regimen that included pomalidomide and carfilzomib within 60 days of index were excluded, as were patients with pomalidomide or carfilzomib claims pre-index or patients who had key missing data elements (e.g., age).

\section{Line of Therapy Definitions}

Index line of therapy (LOT) was composed of all MM treatments of interest (Appendix, available in online article) observed within 60 days of index and continued until the earliest of the following events was observed: (a) the addition of or switch to a new MM treatment $>60$ days post-index or (b) a gap in therapy of $>90$ days followed by a restart of any MM treatment, including the index therapy. Patients were stratified to an index regimen of either pomalidomide or carfilzomib.

\section{Patient Characteristics}

Demographic and clinical characteristics were assessed during the 6-month pre-index period. ICD-9-CM codes were used to identify general comorbid conditions of interest that included cardiac arrhythmia, cardiac valvular disease, chronic pain/ fibromyalgia, diabetes, dyslipidemia, hypertension, myocardial infarction/coronary artery disease, osteoarthritis, renal failure/dialysis, and current or previous smoking. Charlson Comorbidity Index (CCI) scores were calculated excluding MM diagnoses. Medical events related to MM or its treatment were assessed during the 6-month pre-index period and included skeletal-related events (pathologic fracture and spinal cord compression), acute or chronic kidney disease, hypercalcemia, pneumonia, neutropenia, anemia, thrombocytopenia, lymphopenia and/or leukopenia, and peripheral neuropathy. Medical events of interest observed on inpatient claims were reported separately.

\section{Time to Next Treatment}

Time to next treatment (TTNT) was measured as the time from the start of the index therapy to the start date of a subsequent line of therapy. Kaplan-Meir curves and Cox proportional hazards models were used to evaluate the difference in time from the start of the initial therapy to the start of a subsequent line of therapy between the 2 cohorts. Model covariates included age group, gender, number of novel agents in the index regimen, region, pre-index autologous stem cell transplant (ASCT), and key pre-index comorbidities and concomitant medications (i.e., hypertension, smoking or history of smoking, acute or chronic kidney disease, hematologic comorbidities, and use of anticoagulant medications).

\section{Health Care Resource Utilization}

Health care resource utilization was reported during the initial line therapy, including any treatment-free interval before subsequent lines of therapy (i.e., the period from the start of the initial line until the start of the next line of therapy), and separately for the entire follow-up period (irrespective of LOT). Resource utilization was reported up to the time of progression (separate from utilization that occurred during the entire follow-up period), end of continuous health plan enrollment, or the end of the study period, whichever occurred first. Descriptive statistics of resource counts with comparisons between treatment cohorts were reported. Resource count included the following mutually exclusive categories: pharmacy (all pharmacy drugs used by the patient, with MM therapies reported as a separate subcategory); inpatient services; and outpatient services (with emergency room, physician office, and other [i.e., ancillary, lab, radiology, and surgery] encounters reported as separate categories).

\section{Health Care Costs}

Health care costs were reported during the initial line of therapy, including any treatment-free interval before subsequent lines of therapy and separately for the entire follow-up period (irrespective of treatment progression). Health plan-allowed amounts were reported in total, as well as for the following subcategories: pharmacy (with MM therapies including injectable drugs reported as a separate subcategory); inpatient services; and outpatient services (including emergency room encounters). Per patient per month (PPPM) costs were determined by dividing each patient's total costs accrued during the follow-up period by the number of months ( 30 days) that the patient had continuous enrollment and calculating the group average for all of the individual patient averages. Because of the skewness of the data, medians (including median PPPM metrics) were used to report unadjusted costs, while means were used to report cost data in adjusted analyses. All costs were adjusted to 2014 U.S. dollars using the Medical Care Consumer Price Index for All Urban Consumers. ${ }^{19}$

\section{Statistical Analysis}

Analyses and comparisons were conducted between patients initiating therapy with pomalidomide-based regimens and those initiating with carfilzomib-based regimens. Means, medians, and standard deviations (SDs) were calculated for continuous variables. Categorical variables were summarized as cross tabulations in count (frequency) and percentages. To illustrate how treatment groups differed from each other on measures of interest, univariate analyses were conducted using t-tests or Wilcoxon Rank-Sum tests as appropriate for continuous measures. Categorical variables were analysed using chisquare or Fisher's exact tests. 
Real-World Treatment Patterns, Time to Next Treatment, and Economic Outcomes in Relapsed or Refractory Multiple Myeloma Patients Treated with Pomalidomide or Carfilzomib

\section{FIGURE 1 Study Sample Attrition}

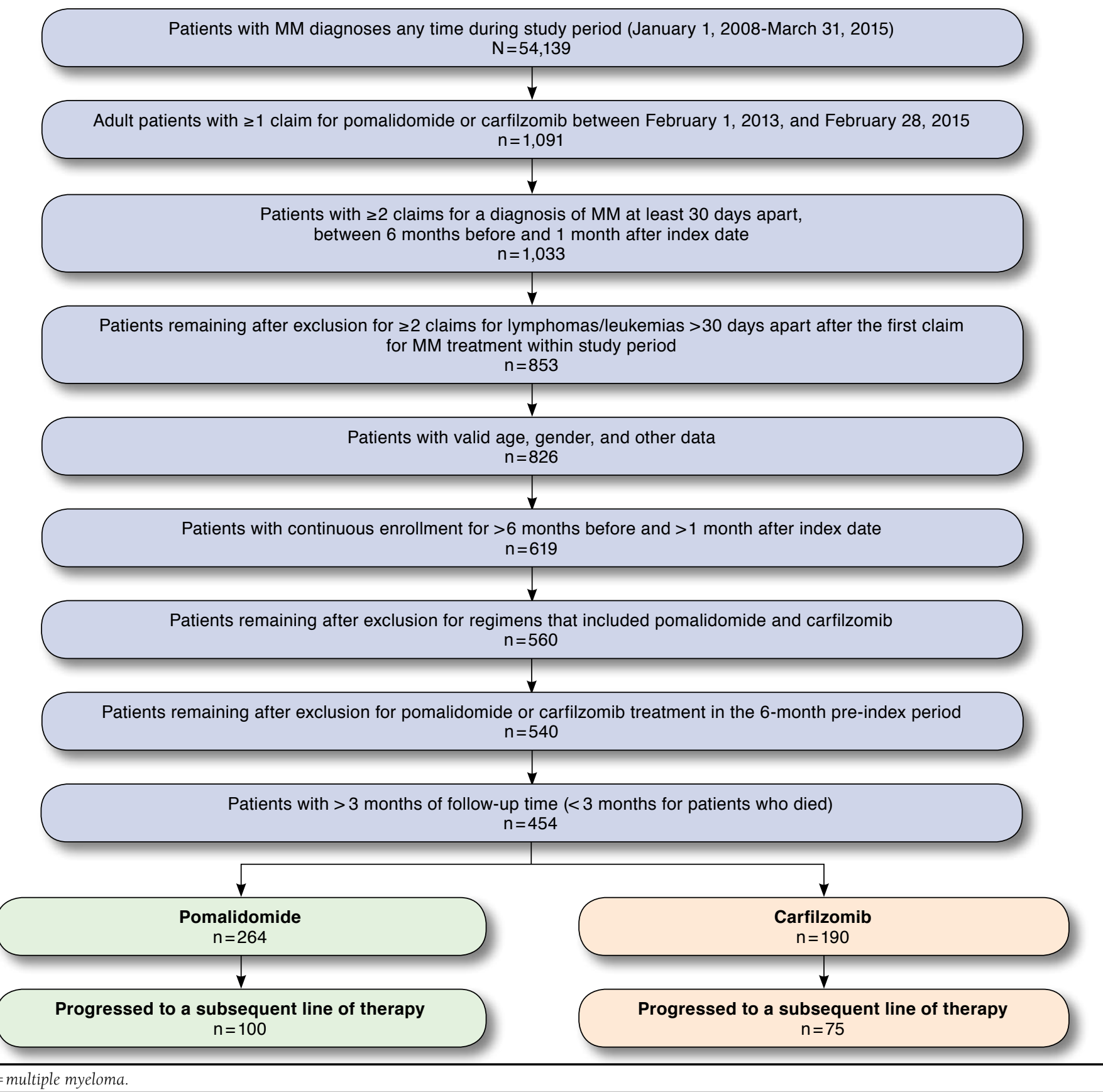

Multivariate generalized linear models (GLM) with a log link function and a negative binomial distribution were used to assess resource utilization where the index line regimen type was tested as the main effect with resource use during the index line as the dependent variable. Models were conducted separately for inpatient and outpatient encounters and adjusted for age group ( $<70$ years vs. $\geq 70$ years); gender; number of novel agents in the index regimen (1 vs. 2 , all others vs. 2); region (Midwest vs. Northeast, South vs. Northeast, West vs. Northeast); pre-index ASCT status; and key pre-index comorbidities and concomitant medications (i.e., hypertension, smoking or history of smoking, acute or chronic kidney 
Real-World Treatment Patterns, Time to Next Treatment, and Economic Outcomes in Relapsed or Refractory Multiple Myeloma Patients Treated with Pomalidomide or Carfilzomib

TABLE 1 Demographic and Clinical Characteristics of Sample

\begin{tabular}{|c|c|c|c|c|c|c|c|}
\hline \multirow{2}{*}{$\begin{array}{l}\text { Characteristic } \\
\text { Age, years, mean [SD] }\end{array}$} & \multicolumn{2}{|c|}{$\begin{array}{l}\text { All Patients } \\
(\mathrm{N}=454)\end{array}$} & \multicolumn{2}{|c|}{$\begin{array}{l}\text { Pomalidomide Patients } \\
\qquad(\mathrm{n}=264)\end{array}$} & \multicolumn{2}{|c|}{$\begin{array}{l}\text { Carfilzomib Patients } \\
\qquad(\mathrm{n}=190)\end{array}$} & \multirow{2}{*}{$\frac{P \text { Value }}{0.671}$} \\
\hline & 61.6 & {$[9.3]$} & 61.7 & {$[9.2]$} & 61.4 & {$[9.6]$} & \\
\hline \multicolumn{8}{|l|}{ Gender, n (\%) } \\
\hline Female & 181 & (39.9) & 110 & $(41.7)$ & 71 & $(37.4)$ & 0.356 \\
\hline Male & 273 & $(60.1)$ & 154 & (58.3) & 119 & $(62.6)$ & \\
\hline \multicolumn{8}{|l|}{ Geographic region, $\mathrm{n}(\%)$} \\
\hline Northeast & 113 & $(24.9)$ & 81 & $(30.7)$ & 32 & (16.8) & 0.007 \\
\hline Midwest & 149 & (32.8) & 76 & $(28.8)$ & 73 & (38.4) & \\
\hline South & 160 & $(35.2)$ & 89 & (3.7) & 71 & $(37.4)$ & \\
\hline West & 32 & $(7.0)$ & 18 & $(6.8)$ & 14 & $(7.4)$ & \\
\hline \multicolumn{8}{|l|}{ Payer type, n (\%) } \\
\hline Commercial & 317 & $(69.8)$ & 188 & $(71.2)$ & 129 & $(67.9)$ & 0.210 \\
\hline Medicare risk & 10 & $(2.2)$ & 7 & $(2.7)$ & 3 & (1.6) & \\
\hline Self-insured group & 119 & $(26.2)$ & 67 & $(25.4)$ & 52 & $(27.4)$ & \\
\hline Other/unknown & 8 & $(1.8)$ & 2 & $(0.8)$ & 6 & $(3.2)$ & \\
\hline Charlson Comorbidity Index score, mean [SD] & 4.2 & {$[2.3]$} & 4.0 & {$[2.2]$} & 4.4 & [2.4] & 0.069 \\
\hline \multicolumn{8}{|l|}{ Baseline comorbid conditions of interest, $n$ (\%) } \\
\hline Acute or chronic kidney disease & 117 & $(25.8)$ & 55 & $(20.8)$ & 62 & $(32.6)$ & 0.005 \\
\hline Anemia & 263 & $(57.9)$ & 143 & $(54.2)$ & 120 & $(63.2)$ & 0.056 \\
\hline Arterial thrombosis & 10 & $(2.2)$ & 6 & $(2.3)$ & 4 & $(2.1)$ & 0.905 \\
\hline Cardiac arrhythmia & 89 & $(19.6)$ & 52 & $(19.7)$ & 37 & $(19.5)$ & 0.953 \\
\hline Cardiac valvular disease & 43 & $(9.5)$ & 24 & $(9.1)$ & 19 & $(10.0)$ & 0.744 \\
\hline Cerebrovascular disease & 10 & $(2.2)$ & 4 & $(1.5)$ & 6 & $(3.2)$ & 0.239 \\
\hline Chronic pain/fibromyalgia & 94 & $(20.7)$ & 50 & $(18.9)$ & 44 & $(23.2)$ & 0.274 \\
\hline Diabetes & 89 & (19.6) & 49 & (18.6) & 40 & $(21.1)$ & 0.509 \\
\hline Dyslipidemia & 123 & $(27.1)$ & 72 & $(27.3)$ & 51 & $(26.8)$ & 0.919 \\
\hline Hypertension & 214 & $(47.1)$ & 117 & $(44.3)$ & 97 & $(51.1)$ & 0.156 \\
\hline $\mathrm{MI} / \mathrm{CAD}$ & 46 & $(10.1)$ & 30 & (11.4) & 16 & $(8.4)$ & 0.305 \\
\hline Neutropenia & 79 & $(17.4)$ & 38 & $(14.4)$ & 41 & $(21.6)$ & 0.046 \\
\hline Osteoarthritis & 213 & $(46.9)$ & 120 & $(45.5)$ & 93 & $(48.9)$ & 0.462 \\
\hline Renal failure/dialysis & 44 & $(9.7)$ & 17 & $(6.4)$ & 27 & $(14.2)$ & 0.006 \\
\hline Smoking or history of smoking & 42 & $(9.3)$ & 19 & $(7.2)$ & 23 & $(12.1)$ & 0.075 \\
\hline Venous thrombosis & 42 & $(9.3)$ & 26 & $(9.8)$ & 16 & $(8.4)$ & 0.605 \\
\hline Pre-index ASCT, n (\%) & 141 & $(31.1)$ & 91 & $(34.5)$ & 50 & $(26.3)$ & 0.064 \\
\hline Available months of follow-up, mean [SD] & 8.9 & {$[5.4]$} & 9.3 & [5.9] & 8.2 & {$[4.5]$} & 0.032 \\
\hline
\end{tabular}

disease, hematologic comorbidities, and use of anticoagulant medications).

Multivariate GLM models with a log link function and gamma distribution were used to compare costs between cohorts where index line regimen type was tested as the main effect. Model covariates were identical to those used in the resource utilization analyses. Separate models were conducted using total costs accrued during the initial line, mean monthly costs accrued during the initial line, and mean monthly costs accrued during the entire eligible post-index follow-up period as dependent variables. CIs around the point estimate for the predicted mean monthly cost of initial line treatment were obtained via bootstrapping based on 500 iterations. Multivariate models of component costs (e.g., pharmacy) were not conducted. All analyses were conducted using SAS version 9.2 (SAS Institute, Cary, NC).

\section{Results}

\section{Patient Demographic and Clinical Characteristics}

A total of 1,091 adult MM patients were identified with $\geq 1$ claim for pomalidomide or carfilzomib between February 1, 2013, and February 28, 2015. After the application of inclusion and exclusion criteria, 454 patients remained for analysis (pomalidomide $n=264$, carfilzomib $n=190$; Figure 1).

Table 1 shows the baseline demographic and clinical characteristics of the sample. The mean (SD) age of patients who initiated therapy with a pomalidomide-based regimen was 61.7 (9.2) years and similar to carfilzomib patients (61.4 [9.6], $P=0.671$ ). Gender was similarly distributed between the pomalidomide and carfilzomib groups with slightly more male patients in each (58.3\% of pomalidomide and $62.6 \%$ of carfilzomib patients, $P=0.365$ ). The mean (SD) follow-up time 
Real-World Treatment Patterns, Time to Next Treatment, and Economic Outcomes in Relapsed or Refractory Multiple Myeloma Patients Treated with Pomalidomide or Carfilzomib

\section{FIGURE 2 Kaplan-Meir Curve Depicting Time from Initial Line of Therapy to Subsequent Line of Therapy (AI} Patients, Irrespective of Observed Treatment Progression)

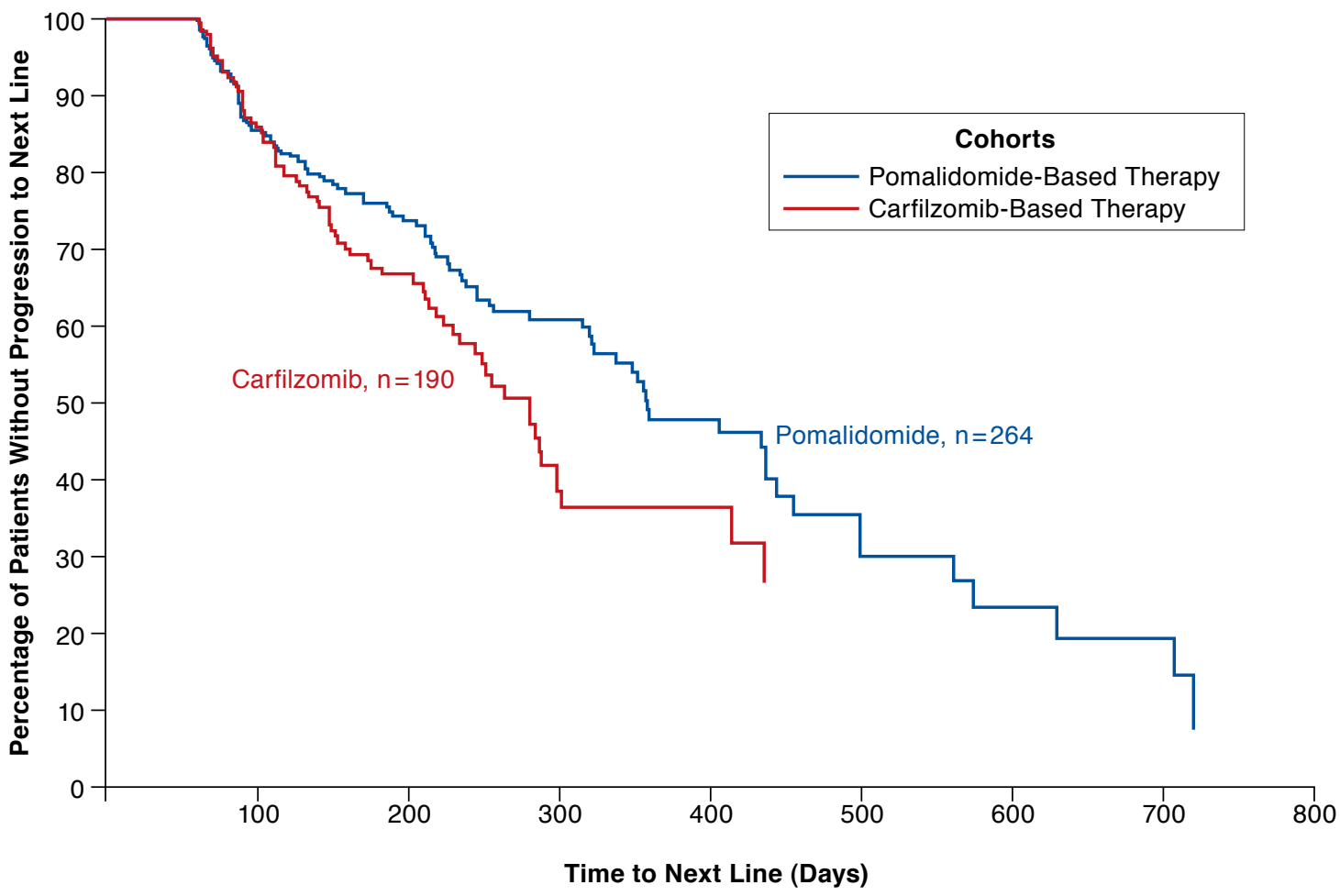

\begin{tabular}{|l|rc|}
\hline \multicolumn{1}{|c|}{ Initial Line of Therapy } & \multicolumn{2}{c|}{ Median (95\% CI) } \\
\hline Pomalidomide-based therapy & 11.9 & $($ (10.7-14.8) \\
\hline Carfilzomib-based therapy & 9.4 & $(7.7-10.0)$ \\
\hline All therapies & 10.7 & $(9.4-13.5)$ \\
\hline
\end{tabular}

\begin{tabular}{|c|c|c|}
\hline Initial Line of Therapy & Log-Rank Test Statistic & P Value \\
\hline Pomalidomide-based therapy vs. carfilzomib-based therapy & 3.5486 & 0.0596 \\
\hline
\end{tabular}

CI= confidence interval; TTNT = time to next treatment.

was 8.9 (5.4) months and approximately 1 month longer in the pomalidomide patient group $(P=0.032)$. At the end of the follow-up period (mean [SD]: 8.9 [5.4] months), 39\% of the sample were on therapy (either the initial line or a subsequent line); $35 \%$ were either not on therapy or died; and approximately $26 \%$ had an unknown treatment status (i.e., there was $<90$ days between the last treatment and the end of follow-up). The most common comorbidities observed during the 6-month pre-index period were hypertension, osteoarthritis, dyslipidemia, chronic pain, arrhythmia, and diabetes, and approximately $60 \%$ of all patients had a history of anemia at baseline. Significantly more carfilzomib patients had a pre-index claim indicative of kidney disease ( $32.6 \%$ vs. $20.8 \%, P=0.005$ ); renal failure or dialysis ( $14.2 \%$ vs. $6.4 \%, P=0.006)$; and/or neutropenia (21.6\% vs. $14.4 \%, P=0.046)$ compared with pomalidomide patients. Pre-index inpatient and outpatient visits for venous thrombotic events and arterial thromboembolic events were similar between the 2 groups (all $P$ values $\geq 0.605$ ) as were hospitalizations for neutropenia (pomalidomide: $6.4 \%$; carfilzomib: $8.9 \% ; P=0.317$ ). The overall mean (SD) CCI score was 4.2 (2.3) and comparable between the 2 cohorts. Pre-index stem cell transplant was observed in $31.1 \%$ of the sample (pomalidomide: 34.5\%; carfilzomib: 26.3\%; $P=0.064$ ).

\section{Treatment Patterns and Clinical Outcomes}

The most frequently observed index regimens for the pomalidomide sample were pomalidomide + dexamethasone (47.0\%) and pomalidomide monotherapy (33.0\%). Nearly half (45.3\%) of 
FIGURE 3 Time to Next Treatment: Initial Line of Therapy to Subsequent Line of Therapy (Patients with $\geq 2$ Lines Observed During Study Period)

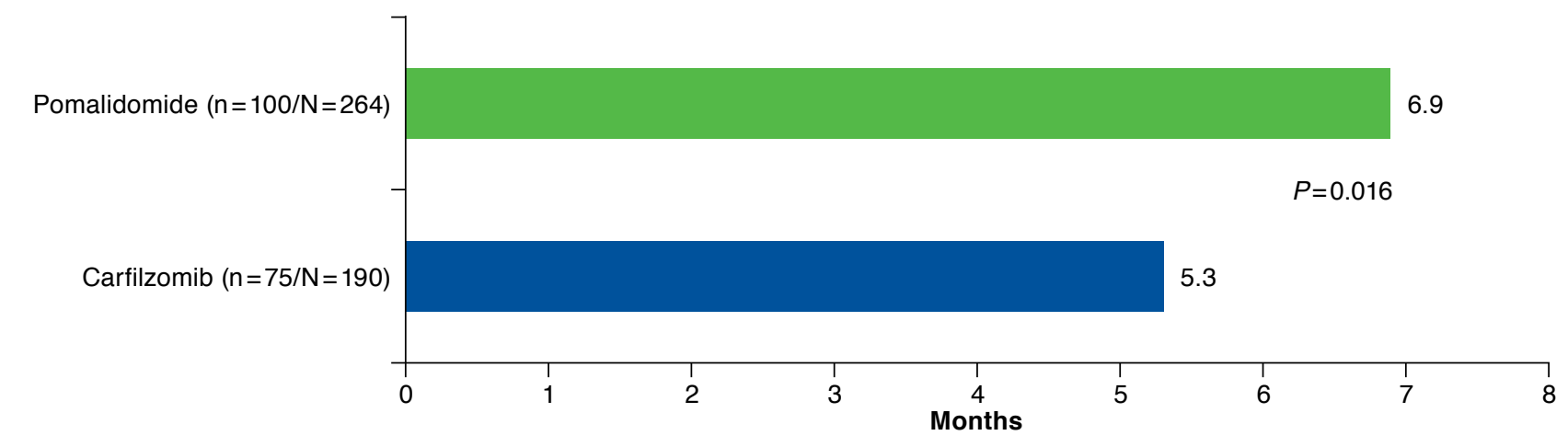

the carfilzomib sample initiated treatment with carfilzomib monotherapy, while the next most frequently observed regimens in the carfilzomib sample were carfilzomib+dexamethasone (14.7\%) and carfilzomib + lenalidomide + dexamethasone (8.4\%).

The mean (SD) duration of initial therapy for pomalidomide patients was 5.9 (4.8) months vs. 4.2 (3.4) months in the carfilzomib sample $(P<0.0001)$. The median $(95 \% \mathrm{CI})$ TTNT for the full sample of pomalidomide patients $(n=264)$ from the Kaplan-Meir analysis was 11.9 (10.7-14.8) months, compared with 9.4 (7.7-10.0) months in the full carfilzomib cohort ( $n=190, P=0.060$; Figure 2). In the subset of patients that could be followed to a subsequent line of therapy (pomalidomide: $n=100$, carfilzomib: $n=75$ ), the mean (SD) TTNT (i.e., time from the initial line of therapy to the subsequent line of therapy) was 6.9 (5.2) months for pomalidomide patients and 5.3 (2.9) months for carfilzomib patients ( $P=0.016$; Figure 3 ). The most frequently observed line of therapy following the initial line in the pomalidomide sample was the addition of (14.0\%) or switch to (15.0\%) carfilzomib \pm dexamethasone (total: 29.0\%). The most frequently observed regimens following the initial line in the carfilzomib sample were pomalidomide + dexamethasone (9.3\%), and carfilzomib monotherapy or carfilzomib + dexamethasone + cyclophosphamide (6.7\% each).

Although a trend towards longer time to progression to a subsequent line was observed in the pomalidomide sample $(P=0.060)$ in the unadjusted analysis, Cox model results, adjusting for key demographic and clinical characteristics, found the difference in the risk of progression to a subsequent line was not significant between pomalidomide and carfilzomib patients ( $\mathrm{HR}=0.862,95 \% \mathrm{CI}=0.616-1.208, P=0.389$ ). None of the covariates included in the model were significant predictors of progression.

\section{Resource Utilization and Economic Outcomes}

Unadjusted analyses of utilization observed during the initial line revealed the proportion of patients with an inpatient visit was similar among patients who initiated therapy with pomalidomide or carfilzomib (49.2\% vs. $54.7 \%, P=0.248$ ), and inpatient visits observed during index line were very low $($ mean $=1)$ for both groups. Pomalidomide patients had fewer outpatient encounters compared with carfilzomib patients (median 116 vs. 173, $P<0.001$ ). There was lower use of pharmacy resources (i.e., all drugs used by the patient regardless of whether they were used to treat $\mathrm{MM}$, including injectable drugs administered in the outpatient setting) by patients whose initial therapy included pomalidomide compared with carfilzomib patients (median number of prescriptions/administrations: 38 vs. $69, P<0.001)$. The median number of pharmacy claims for all MM treatments was also lower in pomalidomide patients compared with carfilzomib patients ( 8 vs. $18, P<0.001$ ).

Inpatient visits were infrequent during the index line of therapy. However, after adjusting for key demographic and clinical characteristics, pomalidomide patients were associated with a higher number of inpatient encounters during the index line of therapy $(P=0.005)$, albeit during a longer index period. The use of $>2$ novel agents in the initial regimen $(P=0.020)$ and the presence of cytopenias during the pre-index period $(P=0.027)$ were significant predictors of inpatient utilization. The total number of outpatient encounters observed during the initial line of therapy was lower in pomalidomide patients compared with carfilzomib patients $(P=0.006)$. The use of just 1 novel agent (vs. $>2$ ) in the initial regimen and Northeast residence $(P=0.033)$ predicted lower outpatient utilization during the initial line of therapy.

Figure 4 shows the unadjusted median costs by place of service incurred during the initial line of therapy. Unadjusted median costs incurred during the initial therapy were $\$ 102,805$ 
Real-World Treatment Patterns, Time to Next Treatment, and Economic Outcomes in Relapsed or Refractory Multiple Myeloma Patients Treated with Pomalidomide or Carfilzomib

\section{FIGURE 4 Unadjusted Median Costs Observed During Initial Therapy}

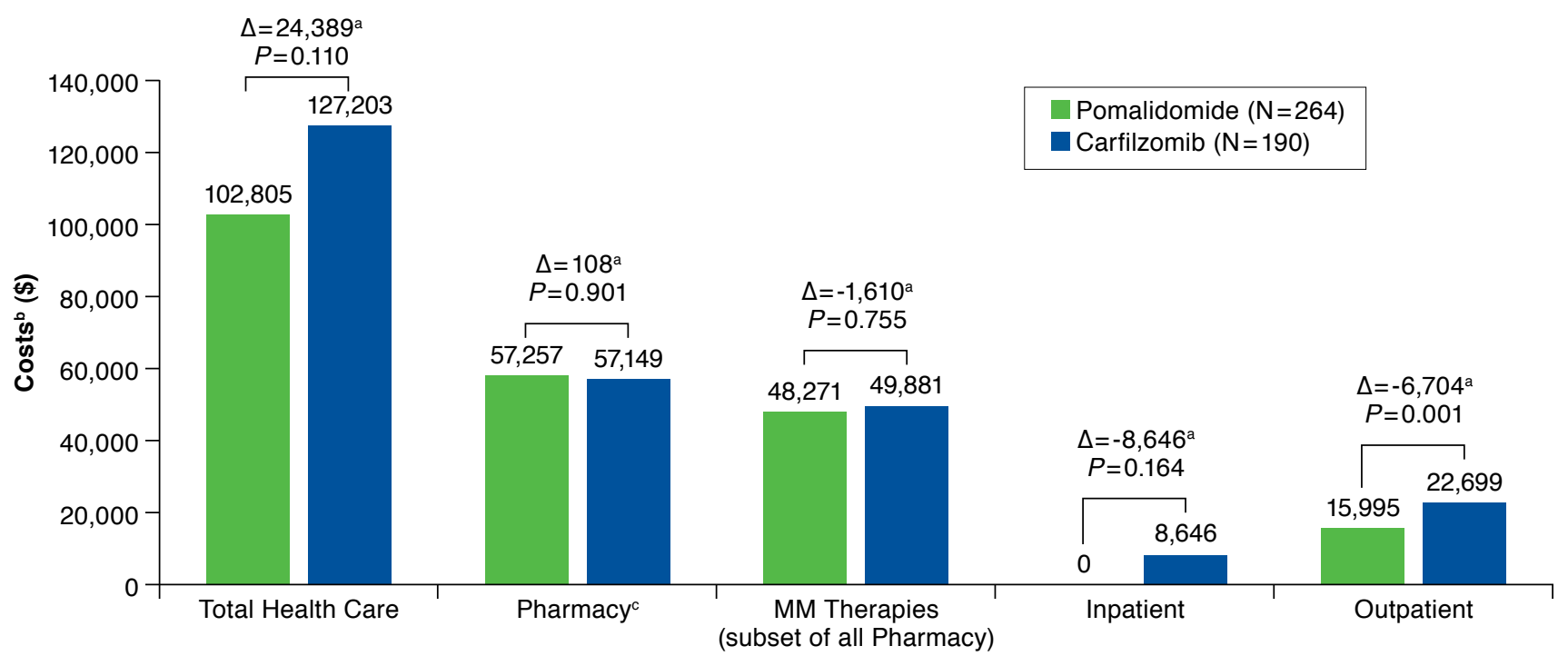

$a_{\Delta}=$ pomalidomide-carfilzomib. Differences in costs and $P$ values were measured with Wilcoxon rank sum tests of medians.

${ }^{b}$ Health care costs were measured over the initial LOT (some patients were still on the initial LOT at last observation).

cPharmacy category includes drugs administered in the outpatient setting.

LOT = line of therapy; $M M=$ multiple myeloma.

for patients initiating treatment with pomalidomide-based regimens and $\$ 127,203$ for patients initiating therapy with carfilzomib-based regimens $(P=0.110)$. Unadjusted median pharmacy costs incurred during the index line of therapy were $\$ 57,257$ for pomalidomide patients and $\$ 57,149$ for carfilzomib patients $(P=0.901)$. MM treatment costs accounted for the majority of pharmacy costs during the index line of therapy in pomalidomide and carfilzomib patients ( $84.3 \%$ and $87.2 \%$, respectively). Unadjusted median inpatient costs incurred during the index line of therapy were $\$ 0$ for pomalidomide and $\$ 8,646$ for carfilzomib patients $(P=0.1638)$; the median number of inpatient encounters were 0 and 1 , respectively. Unadjusted median outpatient costs incurred during the index line of therapy were significantly lower in pomalidomide patients compared with carfilzomib patients ( $\$ 15,995$ vs. $\$ 22,699, P=0.001$ ).

Unadjusted median monthly costs observed during the initial line of therapy were significantly lower in pomalidomide patients compared with carfilzomib patients $(\$ 17,513$ vs. $\$ 24,696, P<0.001)$. Adjusted mean monthly costs incurred during the initial line of therapy remained significantly lower in pomalidomide patients compared with carfilzomib patients $(P=0.013$; Figure 5$)$. Age $<70$ years $(P=0.004)$, male gender $(P=0.016)$, multiple novel agents in the index regimen $(P=0.006)$, pre-index ASCT $(P=0.0484)$, current/previous pre-index smoking status $(P=0.042)$, and pre-index hematologic comorbidities $(P=0.009)$ were all significant predictors of higher mean monthly index line costs. The adjusted mean monthly costs (95\% CI) observed during initial therapy were $\$ 23,888(\$ 21,629-\$ 26,457)$ for pomalidomide and $\$ 27,965$ $(\$ 24,864-\$ 31,524)$ for carfilzomib.

Over the available post-index follow-up period, unadjusted analyses revealed that patients who initiated therapy with carfilzomib-based regimens had a significantly higher median monthly cost $(\$ 24,743$ PPPM vs. $\$ 18,298$ PPPM, $P=0.001)$ compared with pomalidomide-initiated patients. Unadjusted pharmacy $(\$ 11,888$ PPPM vs. $\$ 10,898$ PPPM, $P=0.031)$ and outpatient costs ( $\$ 4,519$ PPPM vs. $\$ 3,009$ PPPM, $P=0.031)$ were also higher in carfilzomib patients compared with pomalidomide patients. Unadjusted inpatient costs were similar between the 2 groups (pomalidomide: $\$ 1,426$ PPPM, carfilzomib: $\$ 2,860$ PPPM, $P=0.074)$. After adjusting for key covariates, pomalidomide-initiated patients trended toward a lower monthly cost over the entire eligible post-index follow-up period, although the finding was not statistically significant $(P=0.230)$. Significant determinants of higher monthly costs included age $<70$ years $(P=0.001)$, male gender $(P=0.018), \geq 2$ novel agents in the index regimen $(P=0.034)$, pre-index ASCT $(P=0.025)$, and pre-index hematologic comorbidities $(P=0.003)$.

\section{Discussion}

This study examined real-world treatment patterns, TTNT, and economic outcomes in MM patients treated with pomalidomide or carfilzomib, using a large U.S. health insurance claims 
Real-World Treatment Patterns, Time to Next Treatment, and Economic Outcomes in Relapsed or Refractory Multiple Myeloma Patients Treated with Pomalidomide or Carfilzomib

FIGURE 5 Adjusted Mean Monthly Costs Observed During Initial Therapy

Pomalidomide patients were associated with $15 \%$ lower monthly initial therapy costs compared with carfilzomib patients.

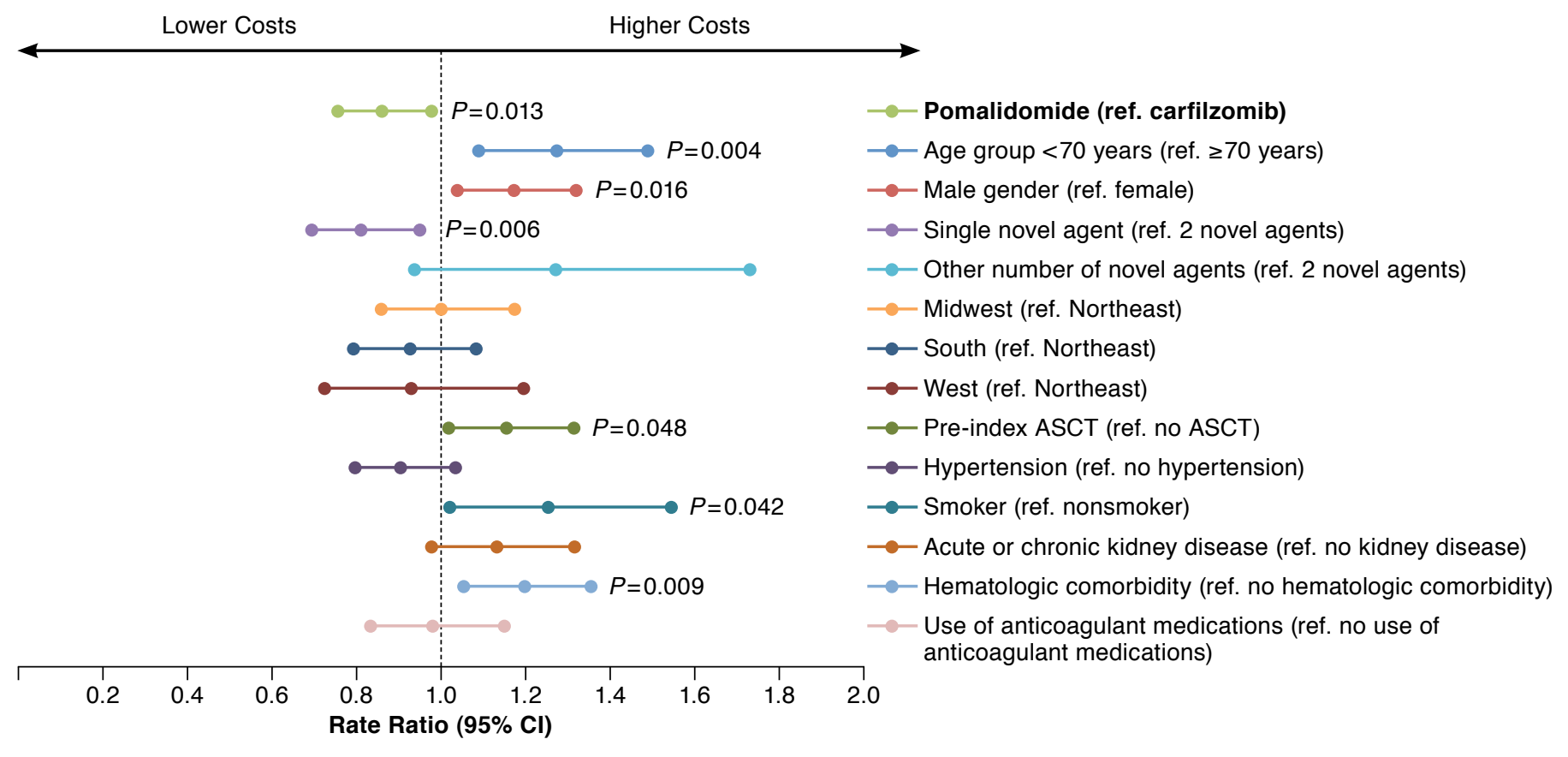

Note: Exponentiated parameter estimates shown represent the multiplicative effect of the variable level compared with the reference level (e.g., pomalidomide compared with carfilzomib). Example interpretations: $0.85=15 \%$ lower costs in pomalidomide patients (compared with carfilzomib patients); $1.18=18 \%$ higher costs in patients with hematologic comorbidity (compared with patients without hematologic comorbidity). The adjusted mean monthly costs (95\% CI) observed during initial therapy were $\$ 23,888$ (\$21,629-\$26,457) for pomalidomide and $\$ 27,965$ (\$24,864-\$31,524) for carfilzomib.

ASCT = autologous stem cell transplant; $C I=$ confidence interval; ref = reference.

database. We believe this to be the first retrospective database study to report TTNT and cost outcomes in a sample of patients with MM treated with pomalidomide or carfilzomib. The estimated median TTNT for the entire pomalidomide sample was higher (11.9 months) than carfilzomib patients (9.4 months); however, both estimates were noticeably higher compared with the PFS of patients treated with similar regimens reported in clinical trials. For example, for pomalidomide + dexamethasone, San Miguel et al. (2013) reported a PFS of 4.0 months and Maciocia et al. reported a PFS of 4.5 months. ${ }^{7,18}$ PFS durations reported for carfilzomib monotherapy range from 3.7 to 8.2 months. ${ }^{11,12,19}$ These differences may reflect differences in regimens (i.e., additional regimens other than those studied in the clinical trials were included in our study), patient populations, and/or methods (e.g., clinically defined PFS vs. TTNT or the PFS proxy used in our study). Thus, the TTNT estimates reported here may not be directly comparable with those from the clinical trials. Nevertheless, our finding provides a real-world comparison that may help inform clinical decisions regarding these therapies for rrMM that were prevalent at the time of the analysis.
Adjusted costs incurred during the initial line of therapy were significantly lower in patients with pomalidomide-based regimens, despite a greater adjusted number of inpatient encounters and a longer TTNT in pomalidomide patients. This cost finding appears to be driven by increased outpatient services associated with carfilzomib treatment, since carfilzomib is typically administered as an infusion in the outpatient setting, whereas pomalidomide is an oral medication requiring fewer outpatient visits. This finding is consistent with the finding of lower unadjusted pharmacy resource utilization in patients whose initial therapy included pomalidomide. The observation of lower unadjusted monthly costs over the entire eligible follow-up period in the pomalidomide group may be related to the longer observed TTNT, since costs escalate upon relapse. This may have led to fewer pomalidomide patients progressing to a subsequent line of therapy during the study period. However, the adjusted monthly costs over the entire eligible follow-up period were not significantly lower in the pomalidomide group.

Overall, this study found that pomalidomide patients trended toward a longer time to progression (TTNT used as a 
proxy measure; statistically significant in the unadjusted measure but not in the adjusted measure), had significantly lower costs during initial therapy, and trended toward lower overall cost when postprogression costs were included. These findings should be interpreted in the context that carfilzomib patients had significantly higher rates of kidney disease/renal failure and neutropenia at baseline. While we were able to control for differences available in the claims data, differences in unavailable characteristics, such as disease staging and severity, may have remained between the 2 groups.

As health systems develop methods to implement episodebased payment systems, there will be a major challenge in benchmarking complex patients, such as the rrMM patients evaluated in this study. The success of these new payment models will rely on real-world data to define episodes, understand the probable range of outcomes using current standards of care, and develop risk-adjustment methods to account for patient populations with varying demographic and clinical characteristics.

As the use of these agents increases over time, additional analysis of larger samples with longer durations of follow-up, as well as more clinical data (e.g., disease severity and staging) may help to validate the results presented here.

\section{Limitations}

The results of this study are subject to a number of limitations. Proxy measures (i.e., TTNT) were used to identify disease progression, since clinical measures such as lab results are not available in claims data. Because changes in treatment may be driven by factors other than disease progression (e.g., tolerability and/or toxicity issues), it is possible that some patients were misclassified as having progressed using TTNT. In addition, the specific number and types of previous lines of therapy could not be determined because patients were not followed from their initial diagnosis and were assumed to have relapsed and/or refractory disease by virtue of observed treatment with pomalidomide or carfilzomib. Song et al. (2016) recently reported little first-line use of pomalidomide and carfilzomib, validating our assumption that these agents are predominantly used in the later line setting. ${ }^{20}$

The PharMetrics Plus claims database is limited to patients with commercial health insurance; therefore, the results may not reflect patients covered by Medicare fee-for-service or Medicaid patients, which may limit the generalizability of the findings. The PharMetrics Plus database does not provide information on systemic factors that could affect care, including benefit and formulary design. However, because of the large and diverse nature of the plans in the database, the effect of these factors on the study results is likely minimal.

Results from observational studies are subject to selection bias and/or coding errors and cannot infer causality. We observed that the majority of each sample initiated treatment with either monotherapy or doublet therapy, including dexamethasone, although the proportion was greater in pomalidomide patients compared with the carfilzomib cohort (pomalidomide \pm dexamethasone: $80 \%$; carfilzomib \pm dexamethasone: $60 \%$ ). It is possible that some retail dexamethasone claims were not captured because of cash payment or other reasons. ${ }^{21}$

Finally, because $17.9 \%$ of carfilzomib-based regimens were composed of carfilzomib in addition to lenalidomide or thalidomide plus or minus a steroid, it is possible that significant heterogeneity among the treatment samples may have influenced the results. Despite these limitations, study strengths include a large sample of patients with robust data regarding clinical and economic outcomes derived from medical and pharmacy insurance claims.

\section{Conclusions}

This retrospective analysis of presumed rrMM patients treated with pomalidomide or carfilzomib-based therapy observed a longer time to a subsequent line of therapy in pomalidomide patients compared with carfilzomib, although the difference was not statistically significant after adjusting for key demographic and clinical characteristics. Compared with patients initiating carfilzomib, pomalidomide patients were observed to have lower adjusted mean monthly costs while on initial therapy and lower unadjusted median PPPM costs over the entire post-index period compared with patients initiated on carfilzomib regimens. These types of analysis may support the development of episode-based payment models. Further analysis with larger sample sizes and a longer follow-up period from the introduction of these new treatment agents is warranted to confirm the findings of this study.

\section{Authors}

CHI-CHANG CHEN, MSPharm, PhD; CATHERINE B. MCGUINESS,

MA, MSc; and ROLIN L. WADE, RPh, MS, QuintilesIMS,

Plymouth Meeting, Pennsylvania. KEJAL PARIKH, MS;

SAFIYA ABOUZAID, PharmD, MPH; LEA PURNOMO, BSc; and MOHAMAD HUSSEIN, MD, Celgene, Summit, New Jersey.

AUTHOR CORRESPONDENCE: Rolin L. Wade, RPh, MS, QuintilesIMS, One IMS Dr., Plymouth Meeting, PA 19382.

Tel.: 908.236.8972; E-mail: rolin.wade@quintilesims.com.

\section{DISCLOSURES}

Funding for this study was provided by Celgene. Chen, McGuiness, and Wade are employees of QuintilesIMS, which was contracted by Celgene to undertake this research. McGuiness also owns stock in Pfizer. Parikh, Abouzaid, Purnomo, and Hussein are employees of Celgene and participated fully in the development and approval of the manuscript.

Portions of the results of this research were previously presented at the American Society of Clinical Oncology (ASCO) 2016 Annual Meeting; June 3-7, 2016; Chicago, Illinois. 
Study concept and design were contributed by Chen, Parikh, Abouzaid, McGuiness, and Wade. Chen and McGuiness took the lead in data collection, along with Wade, and data interpretation was performed by Hussein and Wade, with assistance from the other authors. The manuscript was written by McGuiness, Chen, and Wade, with assistance from Parikh and Abouzaid, and revised by McGuiness and Hussein, along with the other authors.

\section{ACKNOWLEDGMENTS}

The authors thank Kainan Sun and Magdaliz Gorritz, who provided programming and advanced analytical assistance throughout the course of this study, and Kunal Saxena and Katharine Coyle, who provided manuscript writing support on behalf of IMS Health.

\section{REFERENCES}

1. American Cancer Society. What are the key statistics about multiple myeloma? January 19, 2016. Available at: http://www.cancer.org/cancer/ multiplemyeloma/detailedguide/multiple-myeloma-key-statistics. Accessed December 16, 2016.

2. National Cancer Institute, Surveillance, Epidemiology and End Results Program. SEER stat fact sheets: myeloma. Available at: http://seer.cancer.gov/ statfacts/html/mulmy.html. Accessed December 16, 2016.

3. National Cancer Institute. A snapshot of myeloma. November 5, 2014. Available at: http://www.cancer.gov/research/progress/snapshots/myeloma. Accessed December 16, 2016.

4. Song X, Cong Z, Wilson K. Real-world treatment patterns, comorbidities, and disease-related complications in patients with multiple myeloma in the United States. Curr Med Res Opin. 2016;32(1):95-103.

5. Nooka A, Kastritis E, Dimopoulos M. Treatment options for relapsed and refractory multiple myeloma. Blood. 2015;125(20):3085-99. Available at: http://www.bloodjournal.org/content/125/20/3085.long?sso-checked=true. Accessed on December 16, 2016.

6. Shah J, Stadmauer E, Abonour R, et al. Carfilzomib, pomalidomide, and dexamethasone for relapsed or refractory myeloma. Blood. 2015;126(20):2284-90.

7. San Miguel J, Weisel K, Moreau P, et al. Pomalidomide plus low-dose dexamethasone versus high-dose dexamethasone alone for patients with relapsed and refractory multiple myeloma (MM-003): a randomised, openlabel, phase 3 trial. Lancet Oncol. 2013;14(11):1055-66.

8. Pomalyst (pomalidomide capsules). Celgene Corporation. Revised June 2016. Available at: http://www.celgene.com/content/uploads/pomalystpatient-info.pdf. Accessed December 16, 2016.
9. Dimopoulos MA, Palumbo A, Weisel K, et al. Safety and efficacy in the stratus trial, a single-arm phase $3 \mathrm{~b}$ study evaluating pomalidomide low-dose dexamethasone in patients with refractory or relapsed and refractory multiple myeloma. Blood. 2014;124(21):4755-55.

10. Mikhael JR. A practical approach to relapsed multiple myeloma. Hematology Am Soc Hematol Educ Program. 2014(1):262-67.

11. Siegel DS, Martin T, Wang M, et al. A phase 2 study of single-agent carfilzomib in patients with relapsed and refractory multiple myeloma. Blood. 2012;120(14):2817-25.

12. Vij R, Siegel DS, Jagannath S, et al. An open-label, single-arm, phase 2 study of single-agent carfilzomib in patients with relapsed and/or refractory multiple myeloma who have been previously treated with bortezomib. Br J Haematol. 2012;158(6):739-48.

13. Stewart AK, Rajkumar SV, Dimopoulos MA, et al. Carfilzomib, lenalidomide, and dexamethasone for relapsed multiple myeloma. N Engl J Med. 2015;372(2):142-52

14. Elkin EB, Bach PB. Cancer's next frontier: addressing high and increasing costs. JAMA. 2010;303(11):1086-87.

15. The Network for Excellence in Health Innovation. Real-world evidence: a new era for health care innovation. Issue brief. September 2015. Available at: http://www.nehi.net/writable/publication_files/file/rwe_issue_brief_final. pdf. Accessed December 16, 2016.

16. Graf C, Battisti WP, Bridges D, et al. Good publication practice for communicating company sponsored medical research: the GPP2 guidelines. BMJ. 2009;339:b4330.

17. U.S. Bureau of Labor and Statistics. Consumer Price Index seasonally adjusted medical table. Medical Table. Databases, tables \& calculators. March 16, 2016. Available at: https://data.bls.gov/timeseries/ CUUR0000SAM?output_view=pct_12mths. Accessed January 3, 2017.

18. Maciocia N, Sharpley F, Belsham E, et al. Outcome of pomalidomide therapy in relapsed/refractory myeloma: a UK multi-centre experience. Clin Lymphoma Myeloma Leuk. 2015;15(Suppl 3):e288-89.

19. Lendvai N, Hilden P, Devlin S, et al. A phase 2 single-center study of carfilzomib $56 \mathrm{mg} / \mathrm{m} 2$ with or without low-dose dexamethasone in relapsed multiple myeloma. Blood. 2014;124(6):899-906.

20. Song X, Cong Z, Wilson K. Real-world treatment patterns, comorbidities, and disease-related complications in patients with multiple myeloma in the United States. Curr Med Res Opin. 2016;32(1):95-103.

21. Tungol A, Starner CI, Gunderson BW, Schafer JA, Qiu Y, Gleason PP. Generic drug discount programs: are prescriptions being submitted for pharmacy benefit adjudication? J Manag Care Pharm. 2012;18(9):690-700 Available at: http://www.jmcp.org/doi/10.18553/jmcp.2012.18.9.690.

\begin{tabular}{l}
\hline \multicolumn{1}{c}{ APPENDIX Multiple Myeloma Agents of Interest } \\
\hline Bortezomib \\
\hline Carfilzomib \\
\hline Cyclophosphamide \\
\hline Dexamethasone \\
\hline Doxorubicin (liposomal) \\
\hline Lenalidomide \\
\hline Melphalan \\
\hline Pomalidomide \\
\hline Thalidomide \\
\hline Vincristine
\end{tabular}

